\title{
Promotion of formyl peptide receptor 1-mediated neutrophil chemotactic migration by antimicrobial peptides isolated from the centipede Scolopendra subspinipes mutilans
}

\author{
Yoo Jung Park ${ }^{1}$, Sung Kyun Lee ${ }^{1}$, Young Su Jung ${ }^{1}$, Mingyu Lee ${ }^{2}$, Ha Young Lee ${ }^{1}$, Sang Doo Kim ${ }^{1}$, Joon Seong Park ${ }^{3}$, \\ JaeHyung Koo ${ }^{4}$, Jae Sam Hwang ${ }^{5}$ \& Yoe-Sik Bae, , * \\ ${ }^{1}$ Department of Biological Sciences, Sungkyunkwan University, Suwon 16419, ${ }^{2}$ Department of Health Sciences and Technology, SAIHST, \\ Sungkyunkwan University, Seoul 06351, ${ }^{3}$ Department of Hematology-Oncology, Ajou University School of Medicine, Suwon 16499, \\ ${ }^{4}$ Department of Brain and Cognitive Sciences, DGIST, Daegu $42988,{ }^{5}$ Department of Agricultural Biology, National Academy of \\ Agricultural Science, RDA, Wanju 55365, Korea
}

\begin{abstract}
We investigated the effects of two antimicrobial peptides (AMPs) isolated from Scolopendra subspinipes mutilans on neutrophil activity. Stimulation of mouse neutrophils with the two AMPs elicited chemotactic migration of the cells in a pertussis toxin-sensitive manner. The two AMPs also stimulated activation of ERK and Akt, which contribute to chemotactic migration of neutrophils. We found that AMP-stimulated neutrophil chemotaxis was blocked by a formyl peptide receptor (FPR) 1 antagonist (cyclosporin $\mathrm{H}$ ); moreover the two AMPs stimulated the chemotactic migration of FPR1expressing RBL-2H3 cells but not of vector-expressing RBL$2 \mathrm{H} 3$ cells. We also found that the two AMPs stimulate neutrophil migration in vivo, and that this effect is blocked in FPR1-deficient mice. Taken together, our results suggest that the two AMPs stimulate neutrophils, leading to chemotactic migration through FPR1, and the two AMPs will be useful for the study of FPR1 signaling and neutrophil activation. [BMB Reports 2016; 49(9): 520-525]
\end{abstract}

\section{INTRODUCTION}

Neutrophil migration is an important event in the immune response against infection or tissue damage (1). Tissue-resident sentinel cells such as macrophages and dendritic cells recognize and capture invading pathogens, resulting in the activation of the sentinel cells and the production of chemokines

*Corresponding author. Tel: +82-31-290-5914; Fax: +82-31-2907015; E-mail: yoesik@skku.edu

http://dx.doi.org/10.5483/BMBRep.2016.49.9.098

Received 14 June 2016, Revised 18 July 2016, Accepted 8 August 2016

Keywords: Antimicrobial peptide, Chemotaxis, Formyl peptide receptor 1, Neutrophil, Scolopendra subspinipes mutilans
(2). The chemokines cause recruitment of circulating neutrophils in the blood to the infected site $(3,4)$. In addition to chemokines, invading bacteria-derived molecules also cause the recruitment of neutrophils into infected sites $(3,4)$. One important bacteria-derived neutrophil recruiting molecule is formyl peptide (5). As chemotactic neutrophil migration into sites of infection or injury is crucial in regulating immune or inflammatory responses, it is important to identify new molecules that can control neutrophil migration.

Scolopendra subspinipes mutilans has been used in oriental medicine to treat rheumatoid arthritis, lymphadenopathy, and carcinoma $(6,7)$. However, the identities of the bioactive components that regulate the activity of leukocytes or other cell types have not been extensively studied. In a previous report, we identified some antimicrobial peptide (AMP) candidates through de novo RNA sequencing from Scolopendra subspinipes mutilans (8). We demonstrated that some of the AMPs have dual activity, with bactericidal activity and anti-cancer activity against leukemia (9). Recently we also reported that one AMP isolated from Scolopendra subspinipes mutilans stimulates macrophage chemotaxis (10). In this study, we asked whether AMPs isolated from Scolopendra subspinipes mutilans stimulate neutrophil activity. We found that two AMPs from Scolopendra subspinipes mutilans [scolopendrasin III (sequence: VIYVHCTSSRGNNVRLLCAKSKVAP-NH ${ }_{2}$ ), scolopendrasin $\mathrm{V}$ (sequence: YYGGGYKYKHWGCR-NH $\mathrm{N}_{2}$ )], strongly stimulate neutrophil chemotaxis. We also revealed that neutrophil chemotaxis induced by the two AMPs is mediated by an important neutrophil chemoattractant receptor, formyl peptide receptor (FPR) 1.

\section{RESULTS}

Two novel AMPs stimulate neutrophil chemotactic migration in a pertussis toxin (PTX)-sensitive manner

Previously, we and others demonstrated that AMPs, including LL-37 and scolopendrasin VII, stimulate chemotactic migration 
of leukocytes $(10,11)$. In this study, we tested the effects of two new AMPs (scolopendrasin III and scolopendrasin V) isolated from Scolopendra subspinipes mutilans on neutrophil activity. Because the three scolopendrasin AMPs have antimicrobial activity, we compared the amino acid sequence between the three scolopendrasin AMPs using multiple sequence alignment with hierarchical clustering as described previously (12). We found that some amino acids are commonly conserved between two of the AMPs among three scolopendrasin AMPs (Fig. 1A). However, well-conserved amino acids were not detected among the three AMPs (Fig. 1A). We examined the effects of the two new AMPs on neutrophil chemotaxis using a Boyden chamber assay kit. Stimulation of mouse neutrophils with the two AMPs strongly elicited neutrophil chemotactic migration (Fig. 1B). AMPs- induced neutrophil chemotactic migration was concentration- dependent, and maximal activity was induced at $100 \mu \mathrm{g} / \mathrm{ml}$ AMP (Fig. 1B).

Many chemokines and chemoattractants induce neutrophil chemotaxis via PTX-sensitive G-protein(s) $(13,14)$. Therefore, we also investigated whether AMP-induced neutrophil chemotaxis is mediated by PTX-sensitive G-protein(s). Preincubation of neutrophils with PTX prior to the chemotaxis assay with the two AMPs almost completely abolished peptide-induced neutrophil chemotaxis (Fig. 1C). In a positive control experiment, we found that WKYMVm-induced neutrophil chemotaxis was also completely blocked by PTX treatment (Fig. 1C). The results indicate that the two AMPs stimulate neutrophil chemotactic migration via PTX-sensitive G-protein(s).
In addition to chemotactic migration into the infectious or injured area, neutrophils mediate immune responses by stimulating production of superoxide anion (15). We examined whether the two AMPs stimulate superoxide anion production from mouse neutrophils using cytochrome $c$ reduction assay. As shown in Fig. 1D, both AMPs failed to stimulate superoxide anion production. However, the well-known FPR agonist WKYMVm strongly stimulated the production of superoxide anion from neutrophils (Fig. 1D). Degranulation is also important to mediate immune responses against infection (16). The two new AMPs did not stimulate degranulation in neutrophils (Fig. 1E).

\section{Two new AMPs selectively stimulate intracellular signaling} downstream of PTX-sensitive G-protein(s)

There are wide range of extracellular stimuli that elicit diverse intracellular signaling cascades, which mediate various cellular responses. Intracellular calcium increase is an important event, which is associated with neutrophil activation in response to extracellular stimuli (17). In this study, we examined the effects of the two AMPs on calcium signaling in mouse neutrophils. Neither of the AMPs stimulated intracellular calcium increase in neutrophils (Fig. 2A). However, stimulation of mouse neutrophils with WKYMVm strongly elicited intracellular calcium increase (Fig. 2A). ERK is known to play crucial roles in the regulation of neutrophil activity (18). We tested the effects of the two AMPs on the activity of the ERK by monitoring phosphorylation of the enzymes. Stimulation of mouse neutrophils with the two AMPs
A

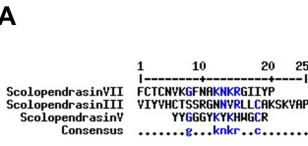

B
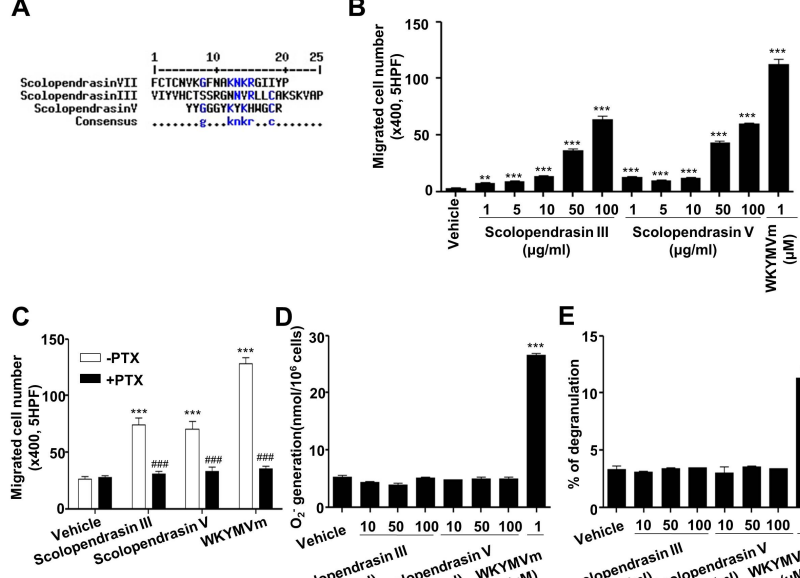

$\mathbf{D}_{\underline{\underline{\underline{T}}}}$

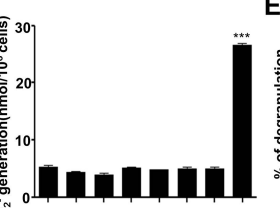

$E$

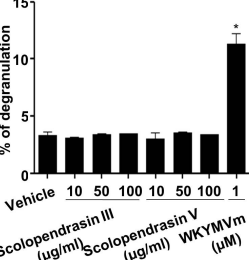

Fig. 1. Two novel AMPs selectively stimulate neutrophil chemotaxis via a PTX-sensitive G-protein(s). (A) Alignment of amino acid sequences of three scolopendrasin AMPs. (B) Mouse neutrophils were applied to the upper well of a multiwell chamber containing several concentrations $(0 \mu \mathrm{g} / \mathrm{ml}, 1 \mu \mathrm{g} / \mathrm{ml}$ $5 \mu \mathrm{g} / \mathrm{ml}, 10 \mu \mathrm{g} / \mathrm{ml}, 50 \mu \mathrm{g} / \mathrm{ml}$, and $100 \mu \mathrm{g} / \mathrm{ml}$ ) of the two AMPs or $1 \mu \mathrm{M}$ of WKYMVm for $90 \mathrm{~min}$. (C) Mouse neutrophils were incubated in the absence or presence of $1 \mu \mathrm{g} / \mathrm{ml} \mathrm{PTX}$ for $4 \mathrm{~h}$, and were applied to the upper well of the multiwell chamber containing $100 \mu \mathrm{g} / \mathrm{ml}$ of the two AMPs or $1 \mu \mathrm{M}$ of WKYMVm for $90 \mathrm{~min}$. The number of migrated cells was determined by counting under a light microscope (B, C). (D) Mouse neutrophils were stimulated with several concentrations $(0 \mu \mathrm{g} / \mathrm{ml}, 10 \mu \mathrm{g} / \mathrm{ml}, 50 \mu \mathrm{g} / \mathrm{ml}$, and $100 \mu \mathrm{g} / \mathrm{ml})$ of the two AMPs or $1 \mu \mathrm{M}$ of WKYMVm and superoxide generated was measured using cytochrome c reduction assay. (E) Several concentrations $(0 \mu \mathrm{g} / \mathrm{ml}, 10 \mu \mathrm{g} / \mathrm{ml}, 50 \mu \mathrm{g} / \mathrm{ml}$, and $100 \mu \mathrm{g} / \mathrm{ml})$ of the two AMPs or $1 \mu \mathrm{M}$ of WKYMVm were treated to mouse neutrophils for $30 \mathrm{~min}$. The peptide-induced secretion of $\beta$ - hexosaminidase was determined. Data are presented as

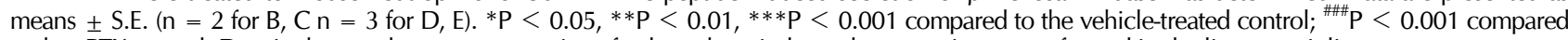
to the-PTX control. Data in the panels are representative of at least three independent experiments performed in duplicate or triplicate. 
A

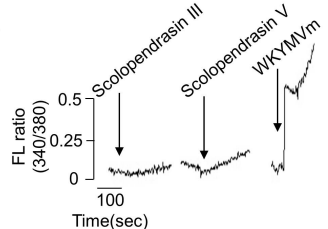

B

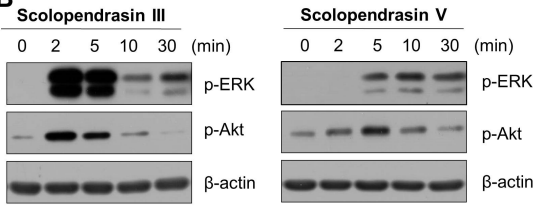

C

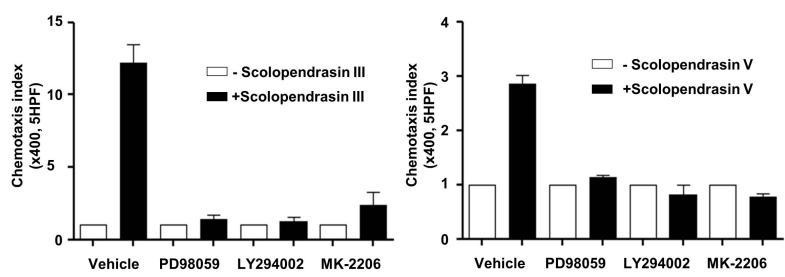

Fig. 2. The two AMPs stimulate ERK and Akt activity in mouse neutrophils. (A) Fura-2 loaded mouse neutrophils were stimulated with scolopendrasin III $(100 \mu \mathrm{g} / \mathrm{ml})$, scolopendrasin V $(100 \mu \mathrm{g} / \mathrm{ml})$, or $1 \mu \mathrm{M}$ WKYMVm, and intracellular calcium levels were determined fluorometrically using spectrofluorophotomter. The peak levels of intracellular calcium were recorded. (B) Mouse neutrophils were stimulated with $100 \mu \mathrm{g} / \mathrm{ml}$ of Scolopendrasin III (left) or Scolopendrasin V (right) for several lengths of time (0 min, 2 min, 5 min, 10 min, 30 min). Total cell lysates were separated SDS- PAGE, and the levels of p-ERK and p-Akt were measured using Western blot analysis. Data are representative of three independent experiments (A, B). (C) Mouse neutrophils were incubated in the absence or presence of PD98059 (50 $\mu \mathrm{M})$ for $60 \mathrm{~min}$, LY294002 (50 $\mu \mathrm{M})$ for $15 \mathrm{~min}$, or MK-2206 $(2 \mu \mathrm{M})$ for $20 \mathrm{~min}$, and were applied to the upper well of a multiwell chamber containing $100 \mu \mathrm{g} / \mathrm{ml}$ of Scolopendrasin III (left) or Scolopendrasin V (right) for 90 min. The number of migrated cells was determined by counting under a light microscope. Data are presented as means \pm S.E. $(n=2)$. Data in the panels are representative of three independent experiments performed in duplicate (C).

markedly induced phosphorylation of ERK (Fig. 2B). Phosphorylation of ERK was transiently induced by the two AMPs, showing time-dependent manner. Scolopendrasin III-induced ERK phosphorylation was transient showing maximal activity at 2-5 min and returning to basal levels at $10 \mathrm{~min}$ (Fig. 2B left). Scolopendrasin V-induced ERK phosphorylation was apparent at 5-30 min after stimulation (Fig. 2B right). Because the two AMPs stimulated chemotactic migration of neutrophils, we tested the roles of ERK on AMP-induced neutrophil chemotaxis using specific inhibitor of ERK (PD98059). Pre-incubation of neutrophils with an ERK inhibitor (PD98059) prior to the chemotaxis assay almost completely inhibited peptide-induced chemotaxis (Fig. 2C). These results indicate that neutrophil chemotaxis induced by the two AMPs is mediated by the activity of ERK.

Akt has also been reported to play a key role in the regulation of cell migration (19). In our study, we tested whether the two AMPs stimulate Akt activity. Stimulation of mouse neutrophils with the two AMPs elicited Akt phosphorylation at 2 to 10 min after stimulation (Fig. 2B). Pretreatment of neutrophils with Akt inhibitor (MK-2206) or phosphatidylinositol 3-kinase (PI3K) (upstream molecule of Akt) inhibitor (LY294002) prior to the chemotaxis assay completely blocked peptide-induced neutrophil chemotaxis, indicating that AMP-induced chemotaxis is mediated by PI3K/Akt pathway (Fig. 2C).

\section{FPR1 mediates neutrophil chemotaxis induced by the two AMPs}

Our finding that the two AMPs stimulate neutrophil chemotaxis, which is completely inhibited by PTX, led us to test the possible role of a well-known neutrophil chemoattractant receptor, FPR1, which is a PTX-sensitive $\mathrm{G}$ protein- coupled receptor. At first we examined the effect of an FPR1 antagonist, cyclosporin $\mathrm{H}(\mathrm{CsH})$ (20), on neutrophil chemotaxis stimulated by the two AMPs. Neutrophil chemotaxis was almost completely blocked by $\mathrm{CsH}$. $\mathrm{CsH}$ also completely inhibited $\mathrm{fMLF}$-induced neutrophil chemotaxis (Fig. 3A). To confirm that the AMP-induced neutrophil chemotaxis is mediated by FPR 1 , we used stably transfected FPR1-expressing RBL-2H3 cells (21). First, we used flow cytometric analysis to confirm that FPR1-expressing RBL-2H3 cells, but not the vector-expressing RBL-2H3 cells, express FPR1 on the cell surface (Fig. 3B). Next, we tested the effect of the two AMPs on chemotactic migration in vector- or FPR1-expressing RBL-2H3 cells. The two AMPs markedly stimulated chemotactic migration of cells in FPR1-expressing but not vector-expressing RBL-2H3 cells (Fig. 3C). The well-known FPR1 agonist $\mathrm{fMLF}$ also strongly induced the chemotactic migration of FPR1-, but not of the vector-expressing RBL-2H3 cells (Fig. 3C). However, neither of the AMPs stimulated the chemotactic migration of cells in the FPR2-expressing RBL-2H3 cells (Fig. 3D). The known FPR2 agonist MMK-1 significantly stimulated the chemotactic migration of FPR2-expressing RBL-2H3 cells (Fig. 3D). These results suggest that FPR 1 but not FPR2 mediates the neutrophil chemotaxis induced by the two AMPs. Since the two new AMPs stimulate neutrophil chemotaxis via FPR1, we also investigated whether the two AMPs induce the chemotactic migration of macrophages, which also express FPR1. As expected, the two AMPs significantly stimulated the chemotactic migration of macrophages (Fig. 3E). 
The two AMPs elicit neutrophil recruitment in vivo via FPR1 Since we observed that the two AMPs stimulate neutrophil chemotaxis in vitro, we then tested their effect on recruitment of neutrophils in vivo. Vehicle administration did not induce neutrophil recruitment into the peritoneal cavity, and no significant difference was observed in either WT or FPR1-deficient mice (Fig. 4A). Administration of the two AMPs into the peritoneal cavity strongly induced recruitment of neutrophils into the peritoneal cavity (Fig. 4B, 4C). Because we found that the two AMPs stimulate neutrophil chemotaxis through FPR1 at the cellular level, we also examined the functional role of FPR1 on AMP-induced in vivo neutrophil recruitment using FPR1-deficient mice. Neutrophil recruitment into the peritoneal cavity induced by the two AMPs was
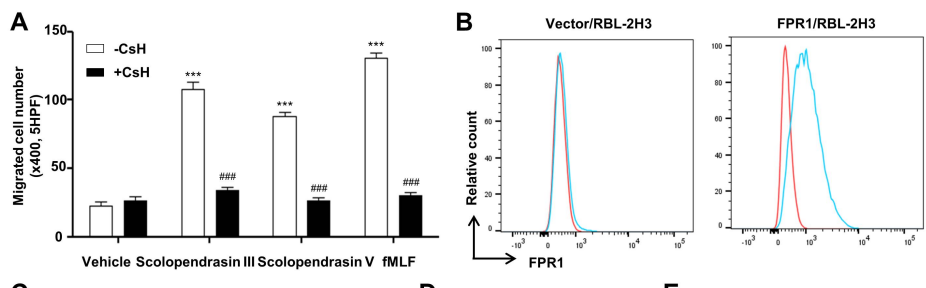

C
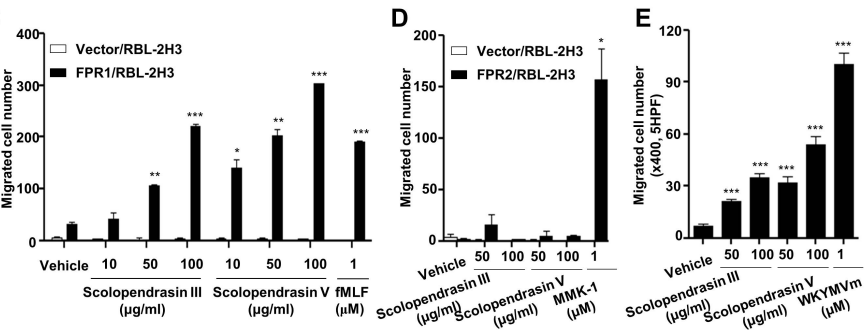

Fig. 3. Neutrophil chemotaxis stimulated by the two AMPs is mediated by FPR1. (A) Mouse neutrophils were incubated in the absence or presence of $\mathrm{CsH}(10 \mu \mathrm{M})$ for $30 \mathrm{~min}$, and were applied to the upper well of a multiwell chamber containing $100 \mu \mathrm{g} / \mathrm{ml}$ of the two AMPs or $1 \mu \mathrm{M}$ of fMLF for 90 min. (B) Cell surface expression of FPR1 was determined by flow cytometric analysis (red, isotype control; blue, anti-FPR1). The results are representative of three independent experiments. (C) Vector- or FPR1-expressing RBL-2H3 cells were applied to the upper well of a multiwell chamber containing several concentrations $(0 \mu \mathrm{g} / \mathrm{ml}, 10 \mu \mathrm{g} / \mathrm{ml}, 50 \mu \mathrm{g} / \mathrm{ml}$, and $100 \mu \mathrm{g} / \mathrm{ml}$ ) of the two AMPs or $1 \mu \mathrm{M}$ of fMLF. (D) Vector- or FPR2-expressing RBL-2H3 cells were applied to the upper well of a multiwell chamber containing several concentrations $(0 \mu \mathrm{g} / \mathrm{ml}, 50 \mu \mathrm{g} / \mathrm{ml}$, and $100 \mu \mathrm{g} / \mathrm{ml}$ ) of the two AMPs or $1 \mu \mathrm{M}$ of MMK-1. (E) Mouse bone marrow-derived macrophages were applied to the upper well of a multiwell chamber containing several concentrations $(0 \mu \mathrm{g} / \mathrm{ml}, 50 \mu \mathrm{g} / \mathrm{ml}$, and $100 \mu \mathrm{g} / \mathrm{ml})$ of the two AMPs or $1 \mu \mathrm{M}$ of WKYMVm for $2 \mathrm{~h}$. The number of migrated cells was determined by counting under a light microscope (A, C, D, E). Data are presented as means $+\mathrm{S}$.E. $(\mathrm{n}=2) * \mathrm{P}<0.05 * * \mathrm{P}<$ $0.01,{ }^{* * *} \mathrm{P}<0.001$ compared to vehicle-treated (A, E) or vector/RBL2H3 cells (C, D); ${ }^{\# \#} \mathrm{P}<0.001$ compared to the AMP alone control (A). Data in the panels are representative of at least three independent experiments performed in duplicate (A, C, D, E).

A

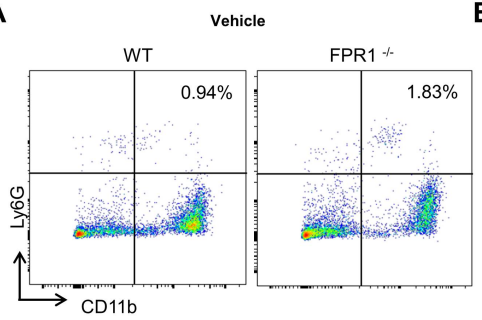

C

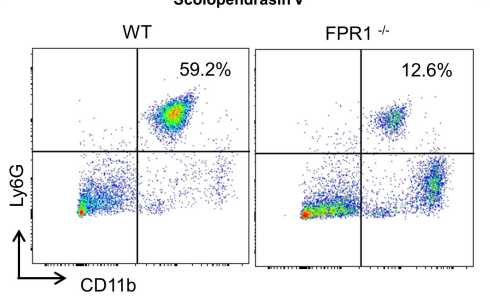

B

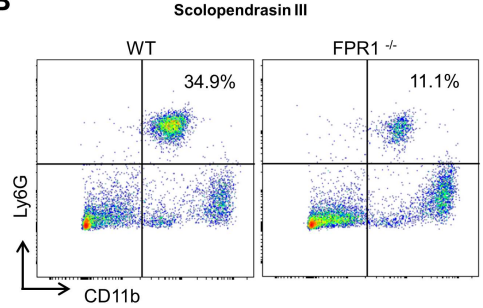

D

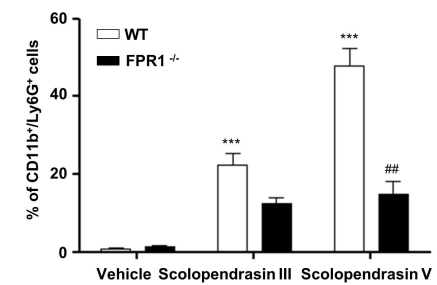

Fig. 4. The two AMPs stimulate neutrophil migration in vivo via FPR1. (A) Vehicle, (B) scolopendrasin III (15 mg/kg), or (C) scolopendrasin $\mathrm{V}(15 \mathrm{mg} / \mathrm{kg})$ were administrated into the peritoneal cavity of WT or FPR1-deficient mice for 2 h. Peritoneal fluid was harvested and recruited neutrophils were analyzed using FACS. Data in the panels are representative of at least three independent experiments. (D) Relative percentages of $\mathrm{CD}_{11 \mathrm{~b}^{+}} \mathrm{Ly}_{6 \mathrm{G}}{ }^{+}$cells from peritoneal fluid were quantified. Data are presented as means \pm S.E. $(n=3-6)$. $* * * P<$ 0.001 compared to vehicle- treated; ${ }^{\# \#} \mathrm{P}<0.01$ compared to WT control. 
markedly attenuated in FPR1-deficient mice (Fig. 4B-D). These results indicate that in vivo recruitment of neutrophils toward the two AMPs is mediated by FPR1.

\section{DISCUSSION}

In this study, we demonstrated that two novel AMPs stimulate neutrophil chemotactic migration, which is an important part of the innate immune system. We found that neutrophil migration stimulated by the two AMPs is mediated by FPR1 in vitro and in vivo. Since the AMPs stimulated neutrophil chemotaxis in FPR1-expressing RBL2H3 cells but not in FPR2-expressing RBL-2H3 cells (Fig. 3C and 3D), the two AMPs seem to be FPR1-selective agonists. Several previous reports demonstrated that various peptide agonists derived from hosts as well as bacteria bind to FPR1, resulting in the activation of leukocytes $(22,23)$. Such agonists include several formyl peptides and annexin $1(23,24)$. However, evidence of AMP binding to FPR1 is limited. We previously demonstrated that one AMP derived from Scolopendra subspinipes mutilans, Scolopendrasin VII, binds to FPR1, resulting in macrophage chemotactic migration (10). Although the concentrations needed to activate FPR1 are extremely high for the two new AMPs compared with the already known FPR1 agonists such as WKYMVm (Fig. 1), our finding on the selective activation of FPR1 by the two other AMPs leading to neutrophil chemotaxis extends the concept that FPR1 is involved in the effect of AMPs on the activation of phagocytic leukocytes, such as macrophages and neutrophils.

Based on our previous report (10) and current finding on the action of two scolopendrasin AMPs (scolopendrasin III and scolopendrasin $\mathrm{V}$ ), we compared their primary sequences. As shown in Fig. 1A, we could not detect any well-conserved amino acids among the three scolopendrasin AMPs. None of the three scolopendrasin AMPs has any amino acid sequences of the two well-known FPR1 agonists (fMLF or WKYMVm). The results suggest that other unrevealed factors other than the primary amino acid sequences might be involved in the binding of the AMPs to FPR1. Because the scolopendrasin AMPs were identified by analyzing the physiochemical properties including length ( $\leq 50$ mer), $\mathrm{pl}(8 \leq \mathrm{pl} \leq 12)$ and positive charge (8), further studies are needed whether or not these characteristics are essential for the binding of the AMPs to FPR1.

Since neutrophils play an important role in host immune defense by generating reactive oxygen species (e.g. the superoxide anion) and releasing granule contents, we tested the effects of the two novel AMPs on the generation of the superoxide anion and degranulation. Although the two AMPs strongly increased chemotactic migration of neutrophils, they did not stimulate superoxide anion production; however, a well-known neutrophil stimulant, WKYMVm, strongly increased superoxide anion production from neutrophils (Fig. 1C). The two AMPs also failed to induce degranulation from neutrophils (Fig. 1D). Many FPR1 agonists have been reported to stimulate intracellular calcium increase, mediating diverse cellular responses $(25,26)$. We investigated whether the two AMPs stimulate intracellular calcium increase using Fura-2/AM, a calcium binding dye. Stimulation of fura-2/AM-loaded neutrophils with the two AMPs did not increase intracellular calcium levels (Fig. 2A). However, a well-known neutrophil stimulant, WKYMVm, strongly induced intracellular calcium increase in the cells (Fig. 2A). Taken together, our results show that the two novel FPR1 agonists stimulate PTX-sensitive G protein/ ERK/Akt pathways, leading to chemotactic migration of neutrophils, without affecting intracellular calcium release and subsequent degranulation and superoxide anion production from neutrophils. In a previous report, we demonstrated that FPR1 is differentially activated by different agonists in a ligand- selective manner (21). Unlike WKYMVm, the WKGMVm and WKRMVm stimulated phosphorylation of ERK/Akt and the subsequent chemotactic migration, but did not stimulate calcium increase and subsequent degranulation activity in FPR1-expressing RBL-2H3 cells (21). Considering that superoxide anion production and degranulation are mediated by intracellular calcium increase in neutrophils $(27,28)$, our finding suggests that the activation of FPR 1 by the two new AMPs do not induce calcium increase-mediated superoxide anion production and degranulation in the cells. The two new AMPs stimulate FPR1, showing selective activation of FPR1 downstream signaling leading to ERK/Akt phosphorylation and subsequent chemotactic migration. Previously, it has been suggested that one G-protein coupled receptor can be activated by different ligands via ligand-selective receptor activation states (29). Binding of different ligands to one G-protein coupled receptor may induce distinct receptor conformation change, resulting in coupling of the receptor to different heterotrimeric G-protein(s), and subsequent activation of different effector enzymes (29). Studies on the detail mechanism involved in the differential activation of FPR1 by the two new AMPs or WKYMVm are needed. In conclusion, in this study we report that the two AMPs (scolopendrasin III and scolopendrasin V) are novel FPR1 agonists. The two AMPs can be used as biased agonists for the selective activation of FPR1 downstream signaling.

\section{MATERIALS AND METHODS}

Materials and Methods are available in the Supplementary Data.

\section{ACKNOWLEDGEMENTS}

This work was carried out with the support of "Cooperative Research Program for Agriculture Science \& Technology Development (Project title: National Agricultural Genome Program, Project No. PJ01033804)" Rural Development Administration, Republic of Korea, and by Basic Science Research Program through the National Research Foundation of Korea (NRF) funded by the Ministry of Science, ICT and future Planning (NRF-2015R1A2A1A10054567). 


\section{REFERENCES}

1. Kolaczkowska E1 and Kubes P (2013) Neutrophil recruitment and function in health and inflammation. Nat Rev Immunol 13, 159-175

2. Heit B, Robbins SM, Downey CM et al (2008) PTEN functions to 'prioritize' chemotactic cues and prevent 'distraction' in migrating neutrophils. Nat Immunol 9, 743-752

3. Wright HL, Moots RJ, Bucknall RC et al (2010) Neutrophil function in inflammation and inflammatory diseases. Rheumatology 49, 1618-1631

4. Craig A, Mai J, Cai S and Jeyaseelan S (2009) Neutrophil recruitment to the lungs during bacterial pneumonia. Infect Immun 77, 568-575

5. Fu H, Karlsson J, Bylund J et al (2006) Ligand recognition and activation of formyl peptide receptors in neutrophils. J Leukoc Biol 79, 247-256

6. Yang M, Xiao C, Wu Q et al (2010) Anti-inflammatory effect of Sanshuibaihu decoction may be associated with nuclear factor-kappa B and p38 MAPK alpha in collagen-induced arthritis in rat. J Ethnopharmacol 127, 264-273

7. Zhao H, Li Y, Wang $\mathrm{Y}$ et al (2012) Antitumor and immunostimulatory activity of a polysaccharide-protein complex from Scolopendra subspinipes mutilans L. Koch in tumor-bearing mice. Food Chem Toxicol 50, 2648-2655

8. Yoo WG, Lee JH, Shin Y et al (2014) Antimicrobial peptides in the centipede Scolopendra subspinipes mutilans. Funct Integr Genomics 14, 275-283

9. Lee JH, Kim IW, Kim SH et al (2015) Anticancer Activity of the Antimicrobial Peptide Scolopendrasin VII Derived from the Centipede, Scolopendra subspinipes mutilans. J Microbiol Biotechnol 25, 1275-1280

10. Park YJ, Lee HY, Jung YS et al (2015) Antimicrobial peptide scolopendrasin VII, derived from the centipede Scolopendra subspinipes mutilans, stimulates macrophage chemotaxis via formyl peptide receptor 1 . BMB Rep 48, 479-484

11. Agerberth B, Charo J, Werr J et al (2000) The human antimicrobial and chemotactic peptides LL-37 and alpha-defensins are expressed by specific lymphocyte and monocyte populations. Blood 96, 3086-3093

12. Corpet $F$ (1998) Multiple sequence alignment with hierarchical clustering. Nucleic Acids Res 16, 10881-10890

13. Porto BN, Alves LS, Fernández PL et al (2007) Heme induces neutrophil migration and reactive oxygen species generation through signaling pathways characteristic of chemotactic receptors. J Biol Chem 282, 24430-24436

14. Spangrude GJ, Sacchi F, Hill HR et al (1985) Inhibition of lymphocyte and neutrophil chemotaxis by pertussis toxin. J Immunol 135, 4135-4143

15. Hampton MB, Kettle AJ and Winterbourn CC (1998) Inside the neutrophil phagosome: oxidants, myeloperoxidase, and bacterial killing. Blood 92, 3007-3017

16. Soehnlein O (2009) Direct and alternative antimicrobial mechanisms of neutrophil-derived granule proteins. J Mol Med (Berl) 87, 1157-1164

17. Futosi K, Fodor S and Mócsai A (2013) Neutrophil cell surface receptors and their intracellular signal transduction pathways. Int Immunopharmacol 17, 638-650

18. Liu X, Ma B, Malik AB et al (2012) Bidirectional regulation of neutrophil migration by mitogen-activated protein kinases.
Nat Immunol 13, 457-464

19. Kim D, Kim S, Koh H et al (2001) Akt/PKB promotes cancer cell invasion via increased motility and metalloproteinase production. FASEB J 15,1953-1962

20. Wenzel-Seifert K and Seifert R (1993) Cyclosporin H is a potent and selective formyl peptide receptor antagonist. Comparison with N-t-butoxycarbonyl-L-phenylalanyl-L-leucylL-phenylalanyl-L- leucyl-L-phenylalanine and cyclosporins A, B, C, D, and E. J Immunol 150, 4591-4599

21. Bae YS, Song JY, Kim Y et al (2003) Differential activation of formyl peptide receptor signaling by peptide ligands. Mol Pharmacol 64, 841-847

22. Schiffmann E, Showell HV, Corcoran BA et al (1975) The isolation and partial characterization of neutrophil chemotactic factors from Escherichia coli. J Immunol 114, 1831-1837

23. Marasco WA, Phan SH, Krutzsch H et al (1984) Purification and identification of formyl-methionyl-leucyl-phenylalanine as the major peptide neutrophil chemotactic factor produced by Escherichia coli. J Biol Chem 259, 5430-5439

24. Walther A, Riehemann K and Gerke V (2000) A novel ligand of the formyl peptide receptor: annexin I regulates neutrophil extravasation by interacting with the FPR. Mol Cell 5, 831-840

25. Forsman H and Dahlgren C (2010) The FPR2-induced rise in cytosolic calcium in human neutrophils relies on an emptying of intracellular calcium stores and is inhibited by a gelsolin-derived PIP2-binding peptide. BMC Cell Biol 11, 52

26. Partida-Sánchez S, Cockayne DA, Monard S et al (2001) Cyclic ADP-ribose production by CD38 regulates intracellular calcium release, extracellular calcium influx and chemotaxis in neutrophils and is required for bacterial clearance in vivo. Nat Med 7, 1209-1216

27. Bréchard S and Tschirhart EJ (2008) Regulation of superoxide production in neutrophils: role of calcium influx. J Leukoc Biol 84, 1223-1237

28. Brown AP and Ganey PE (1995) Neutrophil degranulation and superoxide production induced by polychlorinated biphenyls are calcium dependent. Toxicol Appl Pharmacol 131, 198-205

29. Kenakin T (2001) Inverse, protean and ligand-selective agonism: matters of receptor conformation. FASEB J 15, 598-611

30. Lee SK, Kim SD, Kook M et al (2015) Phospholipase D2 drives mortality in sepsis by inhibiting neutrophil extracellular trap formation and down-regulating CXCR2. J Exp Med 212, 1381-1390

31. Bae YS, Bae H, Kim Y et al (2001) Identification of novel chemoattractant peptides for human leukocytes. Blood 97, 2854-2862

32. Grynkiewicz G, Poenie M and Tsien RY (1985) A new generation of $\mathrm{Ca} 2+$ indicators with greatly improved fluorescence properties. J Biol Chem 260, 3440-3450

33. Lee HY, Kim SD, Shim JW et al (2010) A pertussis toxin sensitive G-protein-independent pathway is involved in serum amyloid A-induced formyl peptide receptor 2-mediated CCL2 production. Exp Mol Med 42, 302-309

34. Gao J-L, Lee EJ and Murphy PM (1999) Impaired antibacterial host defense in mice lacking the $\mathrm{N}$-formylpeptide receptor. J Exp Med 189, 657-662 